\title{
L'Homme
}

Revue française d'anthropologie

165 | janvier-mars 2003

Image et anthropologie

\section{La migration des images}

Histoire de l'art et cinéma documentaire

Denis Vidal

\section{OpenEdition}

Journals

Édition électronique

URL : http://journals.openedition.org//homme/205

DOI : $10.4000 /$ /homme.205

ISSN : 1953-8103

Éditeur

Éditions de l'EHESS

Édition imprimée

Date de publication : 1 janvier 2003

Pagination : 249-266

ISBN : 2-7132-1779-2

ISSN : 0439-4216

Référence électronique

Denis Vidal, « La migration des images », L'Homme [En ligne], 165 I janvier-mars 2003, mis en ligne le

27 mars 2008, consulté le 19 avril 2019. URL : http://journals.openedition.org//homme/205 ; DOI :

10.4000/lhomme.205

〔c École des hautes études en sciences sociales 


\title{
La migration des images Histoire de l'art et cinéma documentaire
}

\author{
Denis Vidal
}

LES ARTS p roches de la propagande présentent un paradoxe pour l'histoirede l'art. Contrairement à ce que l'on pourrait penser, la difficulté n'est pas liée au fait que les problématiques issues de cette discipline ne s'appliqueraient pas à la propagande ; le problème est plutôt qu'elles s'y appliquent trop bien. Cela est particulièrement vrai en ce qui concerne le cinéma documentaire auquel cet a rticle est plus spécifiquement consacré. Cela peut être, en effet, une quête fascinante de chercher les significations religieuses ou politiques implicites dans des fresques de Ghirlandaio et de Pi e ro della Francesca, dans la «tempête " de Giorgione ou la "kermesse" de Rubens ${ }^{1}$. Mais il y a un mérite assurément moindre à dévoiler le contenu politique ou économique de films documentaires qui ont été ouvertement financés dans un but de propagande idéologique ou commercial.

Il est vrai qu'après la Seconde Gu e r re mondiale, Lena Riefensthal aurait certainement préféré qu'on jugeât ses documentaires de l'époque nazie sur des critères purement esthétiques ${ }^{2}$. Mais, dans l'ensemble, la dernière chose que les auteurs de

1. Voir Svetlana Alpers, La Création de Rubens, Paris, Gallimard, 1995 ; Carlo Ginzburg, Enquête sur Pierro della Francesca, Paris, Flammarion, 1981 ; Salvatore Settis, L'Invention d'un tableau, La Tempête de Giogone, Paris, Minuit, 1978 ; Aby Warburg, "L'art du portrait et la bourgeoisie florentine " et "Les dernières volontés de Francesco Sassetti », Essais florentins, Paris, Klincksieck, 1990 : 101-136 ; 167-196. 2. Voir Susan Sontag, "Fascinating Fascism », Under the Sign of Saturn, London, Vintage, 1996 : 73-108.

Cet essai est dédié à Jag Mohan qui s'était attribué le rôle d'historien officiel du cinéma documentaire indien et qui était intarissable sur ce sujet. J'ai aussi beaucoup appris en ayant eu la chance de rencontrer Fali R. Bilimoria et N. S Thapa à Bombay, Mulk Raj Anand à Delhi ainsi que Jehangir Bhownagary à Paris. Tous quatre ont été acteurs et témoins des débuts du cinéma documentaire en Inde. Les premiers résultats de cette recherche ont été présentés, à la conférence sur Warburg à l'ENS, au séminaire de Nalini Delvoye à l'École pratique des hautes études, à une conférence organisée par l'équipe "Identité et mondialisation " de l'IRD à Bondy et au séminaire général du Département d'anthropologie à Cambridge. Les réactions des personnes présentes à ces différentes occasions m'ont aidé à mieux préciser mes idées comme l'ont fait les lectures et critiques de ce texte faites par Carlo Severi, Alexis Tadié et Yves Goudineau. 
films documentaires désiraient était qu’on assimile leur activité à de l'art pur. Et ils auraient été aussi les derniers à dénier les implications idéologiques ou économiques de leurs films. C'était, au contraire, en mettant systématiquement l'accent sur de telles implications qu'ils cherchaient à se faire financer. De même, ils n'étaient pas seulement conscients des contraintes exercées sur eux par leurs commanditaires, ils portaient une égale attention aux réactions du public et à ce qui les motivait. Ainsi, de manière générale, les auteurs de films documentaires s'intéressaient-ils à des questions dont les meilleurs historiens de l' a rt avaient précisément découvert l'importance à la même époque.

Le rôle joué par les arts associés à la propagande, au cours du siècle dernier, ne peut cependant être compris si l'on cherche seulement à les interpréter par rapportau contexte social et culturel pour en déterminer le style, le contenu ou la finalité, qu'il s'agisse de la Révolution soviétique, de la montée du fascisme, de la défense des empires coloniaux ou de celle de la démocratie. Leur histoire est faite, tout autant, demprunts et de ruptures de style, de contenus idéologiques ambigus ou contradictoires, de finalités mal définies et de relations de patronage équivo ques : rien en somme qui corresponde véritablement à l'image traditionnelle que l'on se fait de la propagande.

Mais c'est aussi la raison qui explique de manière paradoxale que les arts proches de la propagande transcendent largement, en fin de compte, toute idéologie précise et tout milieu social bien défini, définissant une géographie culturelle aussi bien qu'une histoire et une sociologie tout à fait spécifiques que l'on ne peut véritablement réduire à aucune autre.

C'est, en tout cas, ce que je voudrais argumenter dans ce bref essai, en montrant comment la tradition du cinéma documentaire de propagande s'est développée dans le monde à partir des années 20 , en me basant plus spécifiquement sur la recherche que je suis en train de mener sur les expressions culturelles de la modernité dans l'Empire britannique et en Inde.

\section{Histoire de l'art et propagande}

Il ne faut pas prend retrop au sérieux Kenneth Clark, le célèbre historien de l' a rt britannique, quand il faisait remarquer que ni ses compétences personnelles ni son activité de directeur de la National Galle ry ne le prédisposaient particulièrement à prendre en charge la section du ministère de l' Information (MoI) chargée d’organiser la propagande cinématographique de la Grande-Bretagne pendant la Seconde Guer re mondiale 3 . Comme il l'explique avec une certaine ironie, non seulement il ne connaissait rien au cinéma mais il ne lisait pratiquement jamais les journaux. La seule raison pour laquelle on devait l'avoir choisi à ce poste était

3. Sur l'histoire du Ministry of Information, et plus particulièrement sur la propagande cinématographique en Angleterre pendant la période de la Deuxième Guerre mondiale, voir en particulier : James Chapman, The British at War, Cinema: State and Propaganda, 1939-1945, London, Tauris Publishers, 1998. 
probablement liée à une confusion sémantique: le même terme de "picture " n'était-il pas indifféremment employé pour désigner un film ou un tableau ${ }^{4}$ !

Il n'est pas surprenant que Kenneth Clark ait pu manifester une certaine ambivalence vis-à-vis du rôle qu'il accepta quand même de jouer au ministère de l'Information ${ }^{5}$. Un tel sentiment fut en fait partagé par la majorité des intellectuels qui participèrent à l'action de cette institution, comme en témoignent, parfois avec génie, des auteurs comme Evelyn Waugh, Graham Greene, et bien entendu, George Orwell ${ }^{6}$. Mais il n'y a pas de doute que son opinion à ce sujet ait divergé sensiblement de celle d'Aby Warburg, en dépit du fait qu'il ait toujours reconnu sa dette intellectuelle envers ce dernier (il se plaisait même à souligner que l'une des conférences d' Aby Warburg à laquelle il avait assisté, sans y comprendre grandchose, d'ailleurs, à cause de sa mauvaise connaissance de l'allemand, avait néanmoins changé d'une manière définitive son approche de l'histoire de l'art).

Aby Warburg, pour sa part, était totalement convaincu en effet de l'applicabilité des enseignements de l'histoire de l' a rtà la propagande. Il avait même édité en 1914 et au début de 1915 quelques numéros d'un magazine illustré (Rivista) dont le but explicite était de renforcer l'alliance entre son pays et l'Italie ${ }^{7}$. Et il se plaisait à inventer des slogans ou des images allégoriques qu'il essayait ensuite de placer auprès des éditeurs de journaux allemands pour qu'ils les utilisent dans leur propagande contrel'Angleterre ou les États-Unis. Son plus grand re g ret, durant la Première Gu er remondiale, était de ne pas avoir disposé d'occasions plus décisives de mettre ses compétences d'historien de l'art au service de l'Allemagne.

Warburg était particulièrement fier d'un slogan de son invention "Britannia rules the slaves». Il y a là une certaine ironie quand on sait que la bibliothèque qu'il avait créée - et à laquelle il tenait par dessus tout - n'avait pu être sauvée des nazis dans les années 30 que grâce à son transfert à bord de deux péniches qui traversèrent la mer du Nord pour accoster à Londres. $s^{8}$ Mais il ne faudrait pas cro i re pour autant qu' Aby Warburg ait été le seul historien de l'art à s'impliquer d'aussi prés dans la propagande de son pays. Le ton employé à la même époque par Émile Mâle, le célèbre historien de l'art français, en apporte peutêtre la meilleure démonstration : «mais il ne s'agira ni d'admiration ni de dénigrement: nous voudrions simplement rassembler des faits qui pro u vent que dans le domaine de l'art, l'Allemagne n'a rien inventé [...]. L'Allemagne avait la prétention d'êt rele grand peuple créateur, il faut lui montrer qu'elle se trompe. Chacun de ceux qui ont étudié un aspect de sa civilisation devrait s'y employer, en attendant que la défaite la remette à son rang qui ne sera pas le premier $"$.

4. Clark Kenneth, The Other Half: A Self-portrait, London, John Murray, 1977: 10.

5. Il fut, en effet, l'auteur, du premier rapport officiel sur la politique à suivre par la Grande-Bretagne pendant la Seconde Guerre mondiale dans le cadre de la propagande cinématographique ; voir Nicholas Pearson, The State and the Visual Arts, Milton Keynes, Open University, 1982: 50.

6. Evelyn Waugh, Put Out More Flags, London, Penguin, 1942; Graham Greene, The Ministry of Fear, An Entertainment, Harmondsworth, Penguin, 1978 (1 $1^{\text {re }}$ éd. 1943); Georges Orwell, Nineteen EightyFour, Harmondsworth, Penguin, 1990 ( $1^{\text {re }}$ éd. 1949).

7. Ron Chernow, The Warburgs, London, Chatto \& Windus, 1993: 176.

8. Ibid. : 406.

9. Émile Mâle, L'Art allemand et l'art fançais du Moyen Âge, Paris, Armand Colin, 1923: 6. 
Pendant l'entre-deux guerres, ce fut également à un historien de l'art (Hans Curlis) que revint un rôle prépondérant dans l'utilisation du cinéma documentaire à des fins de propagande nationaliste en Allemagne, sous couvert de films consacrés à différents thèmes artistiques et culturels. Et si l'on reconnaît aujourd'hui encore une réelle valeur à l'œuvre de Joseph Strzygowski, qui fut le premier historien de l'art à donner toute son importance à l'art des steppes, ce n'en est pas moins avec une certaine gêne, étant donné son implication avec l'idéologie nationaliste puis avec le nazisme ${ }^{10}$. Ce dernier fut d'ailleurs, pour la même raison, une des cibles d'un autre grand historien de l'art français, Pierre Francastel, quand ce dernier rédigea à son tour, en 1940, un livre au ton au moins aussi violent que celui qu’avait publié Émile Mâle lors de la guerre précédente et dont le titre suffit amplement à indiquer la teneur: L'Histoire de l'art, instrument de la propagande germanique ${ }^{11}$.

Peut-être cependant, sur le plan méthodologique, lintervention la plus parad oxale, mais aussi la plus révélatrice des liens entre l'histoire de l'a rt et la propagande, est celle d'Ernst Gombrich. Il faut dire aussi que ce dernier avait ses prop res raisons pour s'in téresser à la question. Il n' a vait pas seulement dû s'exiler d'Autriche sous la menace du nazisme dans les années 30, comme la majorité des historiens de l'art allemands ou autrichiens les plus novateurs de cette période $^{12}$. Une fois arrivé à Londres, il avait passé toute la durée de la guerre à déchiffrer et analyser la propagande hitlérienne pour le compte du même ministère où travaillait Kenneth Clark ${ }^{13}$.

On sait que Gombrich a systématiquement dénoncé toutes les tentatives qui ont pu être faites pour définir une période historique donnée dans des termes que l'on puisse assimiler de près ou de loin à l'esprit du temps. La cible privilégiée de Gombrich est ce qu'il définit comme la conception romantique allemande de l'histoire de l'art, incarnée d'ailleurs plutôt à ses yeux par l'œuvre de Hegel que par celle de Winckelmann ${ }^{14}$. Mais il est prompt à en retrouver les traces jusqu’à la période contemporaine dans des œuvres aussi variées que celles de Burckhardt, de Huizinga ou de Panofsky ${ }^{15}$.

10. Il fut peut-être le premier historien de l'art important à dénoncer systématiquement l'ethnocentrisme, qui continue d'ailleurs plus ou moins de régner dans cette discipline, et à ouvrir ainsi de nouveaux domaines à l'histoire de l'art ; voir Udo Kultermann, The History of Art History, New York, Abaris Books, 1993 : 164-165, voir aussi le jugement assez favorable de E. Gombrich, pourtant peu suspect de sympathie envers le nazisme, sur l'œuvre (sinon sur la personnalité) de Strzygowski, Ernst H. Gombrich, Ce que l'image nous dit. Entretiens sur l'art et la science, Paris, Adam Biro, 1991: 26.

11. Pierre Francastel, L'Histoire de l'art, instrument de la propagande germanique, Paris, Librairie de Médicis, 1945.

12. On assista ainsi en Angleterre, pendant les années 30, à l'immigration d'un nombre considérable d'historiens de l'art (40 au minimum) en provenance des pays sous influence germanique, cf. Barbara Hately-Broad, Refugees Art Historians, Oxford, Brookes University, MA dissertation, 1996.

13. Ernst H. Gombrich, Ideals and Idols, Oxford, Phaidon Press, 1979 : 93.

14. Pour comprendre plus précisément ce que Gombrich entend par une conception hégélienne de l'histoire de l'art, voir Ernst H. Gombrich, "The Father of Art History", in Tributes, Interpreters of our Cultural Tradition, Oxford, Phaidon, 1984: 51-71

15. "In Search of Cultural History», in E. H. Gombrich, Ideals and Idols..., op. cit., 1979 : 24-60. 
Si Gombrich critique avec une telle vigueur toute tendance à identifier le style d'une époque avec ce qu'elle représente plus généralement, partageant de la sorte avec Karl Popper, son ami de toujours, une méfiance profonde à l'égard de toute croyance en une conception globalisante de la culture, ce n'est pas simplement parce qu'il y voit une démarche erronée sur le plan épistémologique ; c'est davantage à cause des dérives idéologiques auxquelles une telle conception peut donner lieu.

On ne saurait douter, en effet, qu'une conception globalisante de la culture ait joué un rôle clé dans la propagande des régimes totalitaires. Et nul n'est prêt à oublier les sinistres connotations qui ont pu être associées au cours du siècle dernier à la mise en place d'un art expressément " socialiste » ou encore authentiquement «national». Mais il n'en demeure pas moins que la réalité est plus complexe que Gombrich semble le considérer.

Si les idéologues des régimes totalitaires croyaient véritablement, en effet, que tout art et toute culture font spontanément écho à ce qui caractérise une époque, pourquoi consacreraient-ils autant d'énergie à la propagande et à la censure ? Et pourquoi, seraient-ils si profondément insatisfaits, non seulement par les formes d'art qu'ils rejettent mais aussi par celles qu'ils ont eux-mêmes choisi le plus souvent de patronner? Paradoxalement, l'accent qui est mis sur la propagande ne témoigne pas seulement d'une conception totalisante de l'art et de la culture; le fait même d'y avoir recours est aussi bien la preuve d'un manque radical de confiance dans toute conception purement " hégélienne » de l'histoire.

Ainsi, pour en re venir au cinéma documentaire, il pourrait sembler que la conception qu' Eisenstein se faisait de son art confirme les analyses de Gombrich quand il insiste, par exemple, dans une lettre ouve rte aux journaux, sur l'impossibilité radicale de produire un film comme Le Cuirassé Potemkine ailleurs qu'en Union soviétique et autrement que sous un régime soviétique ${ }^{16}$. Mais il n'en demeure pas moins, également, que les circonstances qui ont provoqué une telle lettre donnent aux propos d' Eisenstein une signification plus complexe. Ce dernier entendait, en effet, dénoncer, par ce biais, l'appel fait par Goebbels aux cinéastes allemands de s'inspirer directement de ses films pour produire un "Cuirassé Potemkine national-socialiste ${ }^{17}$.

Une telle anecdote illustre clairement, me semble-t-il, les deux versants de l'analyse que l'on doit mener si l'on veut comprendre, non seulement l'importance centrale prise par la propagande dans la premiè re moitié du XXe siècle mais aussi l'é volution de l'histoire de l'a rt à la même époque; et expliquer ainsi, du même coup, la raison pour laquelle les deux domaines ont eu alors tendance à se rapprocher $^{18}$. Si l'on se permet, en effet, de détourner quelque peu la terminologie de

16. "It is only the genuine socialist system of the Soviet Union that is capable of giving birth to the grandiose realistic art of the future and the present", cité par Ronald Bergan, Eisenstein, A Life in Conflict, London, Little, Brown \& Company, $1997: 260$.

17. Ibid.

18. On en a encore une démonstration a posteriori dans les écrits d'Edgar Wind, qui est l'un des historiens de l'a rtle plus proche de Warburg. La lecture de Art and Anarhy, qui fait un peu figure de manifeste dans son œuvre, permet de saisir, en particulier, à quel point certaines façons de s'a pp roprier l'héritage intellectuel de Warburg peuvent contribuer à estomper les distinctions existant sur un plan conceptuel .../... 
Gombrich, on peut alors qualifier le paradigme sous-jacent qui est en jeu dans un tel rapprochement de post-hégélien ou post-romantique. Car ce qui caractérise aussi bien l'histoire de l'art que nombre dautres manifestations intellectuelles, culturelles ou idéologiques de l'époque, ce n'est pas seulement la filiation avec une conception totalisante de l'histoire de l' a rtet de la culture, c'est tout autant la prise de conscience du décalage existant entre une telle conception et la réalité ; c'est aussi, pour certains, la volonté cynique de la pallier par tous les moyens dont ils peuvent disposer. C'est là précisément que la propagande et les arts qui en sont $p$ roches entrent en jeu.

Ainsi, dans un premier temps, prenant comme exemple les débuts du cinéma documentaire en Grande-Bretagne, je montrerai les difficultés spécifiques que l'on rencontre en essayant de mettre en relation la tradition cinématographique que représente le mouvement documentaire anglais avec le contexte qui a été à son origine. Puis, déplaçant alors l'analyse vers le sous-continent indien, je montrerai dans un second temps pourquoi il n'est pas possible, en fait, de rend re compte de ces premières décennies du cinéma documentaire sans élargir le champ de la reche rhe au monde entier.

\section{Harengs saurs et Christmas Pudding}

Les débuts de la tradition documentaire en Grande-Bretagne ont de quoi fairerêver tout historien matérialiste de la culture ${ }^{19}$. Il est rare, en effet, de tro uver une situation où l'on dispose de tous les éléments d'information qui permettent d'établir un lien causal sans ambiguïté entre l'émergence d'un nouveau médium artistique et une série de décisions politiques qui sont elles-mêmes l' e $\mathrm{x}$ pression d'intérêts économiques bien définis. Qui plus est, il n'y a aucune difficulté dans ce cas à observer la "conversion " de différentes variétés de capital entre elles (économique, politique, culturel, familial, etc.) et à proposer, par conséquent, une analyse à la manière de Pier re Bourdieu.

Après la Première Guerre mondiale, et malgré la victoire des alliés, le parti conservateur était particulièrement inquiet de la perte d'influence de la GrandeBretagne dans le monde, qui était due non seulement aux difficultés politiques toujours plus grandes que celle-ci rencontrait dans l'Empire, mais aussi au déclin économique qui en résultait.

Aussi, l'une des premières ambitions de ce parti fut de contrecarrer une telle tendance en cherchant à intensifier par tous les moyens les échanges économiques existant entre les différentes régions del'Empire. Plus précisément, la proposition

entre l'art et la propagande. Non seulement Wind insiste sur le caractère souvent fructueux de l'intervention des commanditaires dans le cours même du processus artistique, mais aussi sur la nécessité de reconnaitre que l'art le meilleur peut avoir un contenu didactique important, et il souhaiterait que les critiques mais aussi les artistes sachent tirer toutes les conséquences d'un tel constat; voir Edgar Wind, Art and Anarchy, London, Faber \& Faber, 1963.

19. Sur les origines de cette tradition, voir en particulier Ian Aitken, Film and Reform : John Grierson and the Documentary Film Movement, London, Routledge, 1990 : chap. 4. 
adoptée lors de la Conférence économique impériale de 1923 fut de transformer prog ressivement celui-ci en une sorte de "marhé commun" où toutes les barrières douanières seraient abolies, tandis que des mesures protectionnistes sévères seraient dressées à ses frontières. Quand Stanley Baldwin parvint au pouvoir en 1924, à la tête d'une coalition politique dominée par les conservateurs, il fut cependant obligé de respecter l'engagement qu'il avait pris au préalable avec ses alliés politiques et renoncer à toute mesure protectionniste. Aussi, pour satisfaire malgré tout leur électorat, les conservateurs durent trouver dautres moyens de manifester leur volonté de renforcer la cohésion de l'Empire. C'est ainsi qu'il en vinrent à créer une nouvelle institution gouvernementale (The Empire Marketing Board) disposant de fonds extrêmement importants et dont la visée exclusive était de pro mo u voir cet objectif en utilisant plus particuliè rement les techniques relat ivement récentes du marketing et de la publicité ${ }^{20}$.

Il faut dire que la passion développée par Rudyard Kipling à la même époque pour le cinéma joua également un rôle important dans les événements que je vo udrais décrire ici. Non seulement celui-ci était considéré alors comme l'un des plus fameux écrivains de l'époque et comme un des idéologues les plus virulents dans la défense de la vocation impériale de la Grande-Bretagne, mais c'était aussi un cousin de Stanley Baldwin. Disposant ainsi - pour suivre encore la terminologie de Pierre Bourdieu - d'une impressionnante variété de capital politique, littéraire ou familial, on ne s'étonnera pas que ce dernier obtint sans difficulté le support de l'Empire Ma rketing Board quand il suggéra de donner au cinéma la place qui lui revenait dans la stratégie de cette institution ${ }^{21}$. Il fut ainsi demandé à Kipling de rédiger le scénario d'un film de propagande en faveur du ren fo rcement des liens commerciaux dans l'Empire. Et pour s'assu rerde sa collaboration, il fut également entendu que la mise en scène en serait assurée par son ami Walter Creighton, qui ne connaissait strictement rien au cinéma, mais avec qui il avait été récemment associé pour organiser l' Exposition impériale de 1924 à Wembley.

Une semaine après que Walter Creighton fut nommé responsable du cinéma au sein de l'Empire Marketing Board, John Grierson, le fondateur du mouvement documentaire anglais (plus connu sous le nom de British Documentary Film Movement ${ }^{22}$ ) revint des États-Unis - et de Chicago, en particulier - où il s'était rendu grâce à une bourse de la fondation Rockefeller. Grierson appartenait, en effet, à la première génération d'étudiants qui s'étaient intéressés aux phénomènes de communication de masse. Après avoir étudié le rôle des journaux

20. Sur le contexte politique de la création de l'EMB par l'un de ses principaux acteurs, voir en particulier Leo Amery, My Political Life, London, Hutchinson, 1953, II : chap. 4.

21. L'ensemble de cet épisode est bien décrit dans Paul Swann, The British Documentary Film Movement, 1926-1946, Cambridge, Cambridge University Press, 1989 : chap. 2, 21-48.

22. Bien qu'il soit généralement moins bien connu en France, le British Documentary Film Movement occupe une place importante dans l'histoire du cinéma qui peut se comparer à celle du Néoréalisme en Italie ou encore à celle de la Nouvelle Vague ou du Cinéma Vérité en France. En dehors de leur intérêt historique et sociologique, un petit nombre de ces documentaires sont d'une réelle qualité cinématographique. Tel est le cas, en particulier, de Night Mail, de Basil Wright et Harry Watt, qui date de 1936 et dont certaines séquences sont de purs chefs-d'œuvre. 
populaires aux États-Unis, il s'était passionné pour les possibilités qu'offrait le cinéma dans le domaine de la propagande. Il s'arrangea aussi, dès son retour en Angleterre, pour être introduit auprès de Stephen Tallents, le directeur de l'Empire Marketing Board. Et il sut le convaincre, sans difficulté, qu'il était l'homme idéal dont ce dernier avait besoin pour prendre en charge la propagande cinématographique de son institution. Mais Tallents s'était déjà engagé auprès de l'ami de Kipling et il ne pensait pas être en mesure d'obtenir aisément l'autorisation administrative dont il avait besoin pour financer deux films en même temps. Stephen Tallents et Grierson ne manquaient cependant pas d'ingéniosité. Or, ils avaient découve rt que le secrétaire gouvernemental, responsable du financement de tels projets, était aussi l'auteur d'un petit chef d’œuv re littéraire intitulé : Le Hareng saur et son impact sur l'histoire del'Angleterre. Le problème put donc être rapidement réglé; et c'est pourquoi le scénario de Drifter, un des plus célèbres films de toute l'histoire du cinéma documentaire, est entièrement axé sur la pêche aux harengs.

Il faut dire que John Grierson bénéficia également d'une seconde chance. Il n'y a pas de doute, en effet, que le film de Kipling et de son ami était un véritable navet. Il s'intitulait Une seule famille et, pour l'essentiel, relatait comment étaient récoltés divers produits agricoles dans l'Empire. Mais, surtout, le point culminant du film était la scène montrant tous les produits récoltés solennellement apportés à Buckingham Palace afin de préparer... un Christmas Pudding pour le roi-empereur. En outre, chaque colonie et chaque dominion de l'Empire étaient incarnés et personnalisés par une femme de la haute société. Je n’ai pas vu ce film, mais il n'est pas surprenant d'apprendre qu'il fut éreinté par la critique et subit un véritable échec, d'autant qu'il avait coûté fort $\operatorname{cher}^{23}$.

En re vanche, le film de Grierson obtint un vif succès, particulièrement lorsqu'il fut présenté pour la première fois à Londres, lors d'une séance de ciné-club à laquelle assistait toute l'intelligentsia de l'époque (en particulier, les membres du cercle de Bloomsbury, comme Virginia Woolf ou Keynes). Il est important de souligner que ce public ne s'était pas seulement déplacé pour voir un film consacré à la pêche aux harengs, Grierson avait, en effet, eu la bonne idée de présenter son prop re documentaire en avant-programme de la séance où était montré, pour la premièrefois en Angleterre, Le Cuirassé Potemkine - jusque-là interdit par la censure mais dont la réputation était déjà considérable - et ce en présence de l'aute ur ${ }^{24}$. Cela valut d'ailleurs à Grierson dêt re considéré à son tour comme une so rte d'Eisenstein anglais. Il est vrai qu'il s'était ouve rtement inspiré du film de celui-ci, qu'il connaissait d'autant mieux qu'il en avait lui-même traduit les soustitres pour sa présentation aux États-Unis.

Il semble en outre, mais il faut être prudent sur ce point, que le film de Grierson ait aussi rencontré un certain succès quand il fut montré à un public

23. Paul Swann, The British Documentary Film Movement..., op. cit., 1989: 35.

24. Voir en particulier le récit de cette séance, in ibid. : 34.

\section{Denis Vidal}


plus large ${ }^{25}$. Il ne fait aucun doute que Tallents a considéré l'expérience réussie. Et cela le convainquit, ainsi que quelques autres fonctionnaires haut placés, que le cinéma documentaire pouvait effectivement devenir un instrument précieux de propagande gouvernementale.

Grierson ne fut cependant pas le seul cinéaste, loin s'en faut, à s'inspirer directement d'un autre cinéaste dont l'œuvre se situait dans un contexte idéologique complètement opposé au sien. Comme on l'a vu précédemment, Goebbels avait exigé la même chose des cinéastes allemands. Et quand Frank Capra fut chargé à son tour de la propagande cinématographique pour le compte de l'armée américaine, ce furent les films de Leni Riefensthal qui lui furent montrés en privé pour qu'il s'en inspirât, alors qu'officiellement ils étaient censurés aux États-Unis. Ainsi assiste-t-on, par le biais de ces influences croisées, à l'émergence d'un nouveau style international qui ne saurait être identifié exclusivement aux intérêts spécifiques d'un pays, d'une idéologie ou d’un régime politique bien déterminé.

Ce qui est fascinant dans l'épisode précédent, c'est qu'il correspond très exactement à la confrontation entre deux des plus grandes traditions de représentation du début du XXe siècle : la première d'entreelles, que personnalise parfaitement le personnage de Kipling, correspond à l'idéologie et à l'esthétique qui dominèrent largement la seconde moitié du XIX siècle, et la seconde, qui incarnera durablement l'idée de modernisme, va s'imposer pratiquement jusqu'à nos jours. Cela ne veut cependant pas dire qu'un tel épisode a été complètement unique en son genre. Mais il n'en est pas moins étonnant de constater qu'un film inspiré par Kipling et qui commémorait ouve rtement la grandeur de l'Empire britannique ait pu être finalement rejeté par des politiciens conservateurs et des fonctionnaires gouvernementaux, alors que ceux-ci n'étaient pas seulement des défenseurs farouches de l'Empire britannique mais aussi des hommes qui jouèrent un rôle clé dans la lutte idéologique, en Grande-Bretagne, contre le socialisme (Stephen Tallents ou Leo Amery, en particulier). Et pourtant, ce seront les mêmes personnes qui seront prêtes à pro mouvoir un créateur ouve rtement inspiré par des films à la gloire de la Révolution bolchevique. Il faut dès lors essayer de compren d re comment interpréter le contenu et la fonction véritable des œuvres qui résultaient d'une telle association?

Il n'y a pas de doute, en effet, que Grierson a été, à son époque, considéré comme un metteur en scène pro g ressiste. Cela était d'ailleurs vrai également de l'ensemble des cinéastes qui constituaient l'école documentariste anglaise même si - comme on peut s'y attendre - tout le monde n'était pasd'accordsur le degré de radicalisme qu'on devait leur prêter. Il ne s'agissait pas seulement d’un jugement complaisant porté sur eux-mêmes par les cinéastes ou les intellectuels associés au Mo uvement, c'était aussi l'opinion des commanditaires de leurs films qui manifestaient parfois leur inquiétude face à ce radicalisme supposé. Grierson n'a pas seulement été suspecté d'êt re trop à gauche, il s'en est fallu de 
peu qu'il soit également accusé d' êt reun espion au service de l'Unionsoviétique pendant la guerre froide ${ }^{26}$.

La réputation de Grierson et de ses collègues n'était pas due seulement au fait qu'ils affirmaient ouve rtement leur admiration pour le cinéma soviétique, mais ils se flattaient également d êt reles premiers cinéastes à montrer réellement les conditions de vie des travailleurs et à leur donner aussi la possibilité de s'exprimer plus ou moins directement à l'écran. Le fait tout aussi indiscutable que leurs films fu rent, comme on l'a vu, essentiellement financés par des politiciens conservateurs, soucieux de défendre des formes d'impérialisme surannées, ou encore par des entreprises multinationales, tout aussi désireuses d'en promouvoir des variantes plus contemporaines, correspondait moins bien évidemment à une réputation de pro g ressisme. Aussi la question est-elle de savoir comment expliquer ce paradoxe qui semble contredire aussi bien la logique de la propagande que celle du patronage et dont Grierson lui-même s'était étonné ?

Il est d'ailleurs frappant de constater, qu'à l'exception de quelques marxistes radicaux, personne ne semble s'être véritablement soucié de cette question jusqu'aux années 80 . On dispose ainsi, encore en 1988, du témoignage d'une spécialiste canadienne des médias décrivant, dans l'introduction du livre qu'elle avait consacré à Grierson, sa stupéfaction et son horreur lorsqu'elle découvrit que ce dernier n'était peut-être pas, après tout, le progressiste qu'elle avait toujours imaginé qu'il était ${ }^{27}$. Du même coup, celle-ci n'eut d'ailleurs de cesse de démontrer dans son livre que Grierson n'était en réalité qu'un valet de l'impérialisme et un propagandiste déguisé.

Il y a eu cependant depuis lors des tentatives à la fois plus subtiles et plus nuancées - comme celle de Brian Winston, en particulier ${ }^{28}$ - pour démontrer que les documentaires anglo-saxons de cette période n'étaient pas tant caractérisés par une intention propagandiste que par un art consommé d'éviter toute question politique de fond. Et c'aurait été précisément ce talent que les commanditaires de ces films auraient apprécié par dessus tout. Il n'y a pas de doute qu'une telle interprétation peut être vraisemblable quand on voit la plupart de ces films aujourd'hui. On peut cependant se demander si, comme la précédente, elle ne souffre pas d'un certain anachronisme.

À bien y réfléchir il n'est pas étonnant que des documentaires consacrés à des sujets tels que le chauffage au gaz ou le fonctionnement des postes ne possèdent pas toujours, lors de leur visionnement aujourd'hui, un contenu politique manifeste. Ce qui est plus surprenant, en re vanche, c'est que leurs auteurs, vo i re leurs commanditaires et leurs critiques, et parfois même leur public, aient pu y déceler non seulement de réelles intentions sociales, mais aussi un progressisme politique sous-jacent.

Il peut bien sûr y avoir des exceptions, mais, dans l'ensemble, on doit prend re acte du fait qu'un grand nombre de ces films documentaires, qui servaient pour-

26. Joyce Nelson, The Colonized Eye: Rethinking the Grierson Legend, Toronto, Between The Lines, 1988 : ch. 9, 153-163.

27. Ibid.

28. Brian Winston, Claiming the Real..., op. cit., 1995 : chap. 12. 
tant de manière explicite à des buts de propagande ou de publicité, n'exprimaient pas de manière aussi claire que l'on pourrait s'y attendre l'idéologie ou les intérêts supposés de leurs commanditaires. Il leur arrivait parfois de les contredire ouvertement ${ }^{29}$. Là encore il ne s'agissait pas d'une situation exceptionnelle. Aussi n'y a-t-il pas de doutes que de tels faits démentent l'image traditionnelle qui est celle de la propagande et de la publicité ; c'est d'ailleurs là tout leur intérêt.

\section{Une carrière variée}

Déplaçons-nous maintenant dans une autre région du monde pour examiner les débuts du cinéma documentaire dans l'Inde indépendante, deux à trois décennies plus tard. Auparavant, je souhaiterais donner quelques indications rapides sur les antécédents de cette tradition cinématographique, alors que le sous-continent indien faisait encore partie de l'Empire britannique. Il est commode, dans cette perspective, de distinguer schématiquement trois périodes avant l'Indépendance de ce pays.

Celle qui débute avec l'arrivée à Bombay en 1896 de Maurice Sestier, un collaborateur de Louis Lumière, une année à peine après que la première séance publique de cinéma se fut tenue dans un café des Grands Boulevards. Pendant les trente années qui suivirent, un grand nombre de cinéastes indiens tourneront des films extrêmement courts - ils duraient généralement quelques minutes seulement - sur divers aspects de la réalité du pays. Mais, en dépit de cela, les cinéastes indiens qui jouèrent un rôle clé dans le développement et l'institutionnalisation d'une véritable tradition documentaire en Inde, au cours des années 30 et 40, semblent avoir été ceux qui étaient revenus, il y a peu, de l'étranger et qui occupèrent une place assez semblable à celle jouée par Grierson en Angleterre, à son retour des États-Unis. Il est aussi remarquable de constater que l'expérience acquise par cette poignée d'individus l'avait été pratiquement dans tous les endroits importants où le cinéma se développait alors dans le monde.

C'est ainsi, par exemple, que D. G. Tendulkar, qui allait devenir le biographe officiel de Gandhi, commença sa carrière comme auteur de films documentaires, formé en Union soviétique auprès d'Einsenstein. K. S. Hirelekar, fondateur de la Motion Picture Society of India, avait, quant à lui, été formé à l'UFA, en Allemagne, qui, avec la tradition des kulturfilms, était un des principaux centres de films documentaires dans le monde. Ezra Mir avait travaillé à Hollywood et apportait de ce fait sa connaissance de la tradition américaine de documentaires

29. Il y a ainsi l'exemple spectaculaire de Joris Ivens, employé par le gouvernement hollandais en 1945, pour tourner un documentaire qui montrerait, sous un jour glorieux, la libération de l'Indonésie par une flotte alliée. Mais après que les événements eurent pris une tournure inattendue, avec la proclamation de l'indépendance par Sukarno, Ivens fit un film (Indonesia Calling, 1946) dont le ton était complètement différent de celui qu'on attendait de lui et qui scandalisa totalement ses commanditaires qui le censurèrent. Le même film fut utilisé, en revanche, comme un outil de propagande par les forces indonésiennes qui combattaient les Anglais et les Hollandais; voir Erik Barnouw, Documentary: A History of the NonFiction Film, New York, Oxford University Press, 1974: 170-171. 
(March of the Time). Entre toutes, il y a une personne que je tiens à mentionner : P. V. Pathy, qui n'a pas seulement été un des cinéastes les plus importants de cette première période du cinéma documentaire en Inde, mais qui, en plus, avait soutenu une thèse de doctorat d'État sur Le Théatre contemporain en Andra Pradesh à la Sorbonne, sous la direction de Sylvain Lévi, avant de se passionner pour le cinéma et suivre un formation de cameraman à Vaugirard ${ }^{30}$.

En dépit de tentatives variées pendant les années 30, le développement d'une véritable tradition de cinéma documentaire dans le sous-continent indien n'en a pas moins été lié essentiellement aux besoins de propagande des Britanniques dans la région pendant la Seconde Guerre mondiale. C'est aussi dans ce cadre que des cinéastes formés par Grierson (Alex Shaw et James Beveridge, en particulier) aidèrent à mettre sur pied une base institutionnelle susceptible de permettre la production régulière de films documentaires avec l'assistance de producteurs et de cinéastes indiens. L'institution créée par le gouvernement impérial (Information Film of India) fut dissoute par Nehru, juste après la Seconde Guerre mondiale et quelques mois seulement avant l'Indépendance, lequel Nehru voyait en celle-ci essentiellement un instrument de propagande au service des Anglais. Mais, à peine un an plus tard, cette organisation fut remise sur pied sur la recommandation de Valabhai Patel, premier ministre de l'Inde indépendante, qui était alors l'homme d'État le plus puissant du pays après Nehru. La raison de ce revirement administratif avait été la découverte tardive faite par le gouvernement indien qu'en l'absence d'une telle institution la couverture cinématographique des cérémonies de l'Indépendance était entièrement laissée à l'initiative de compagnies étrangères.

Une conséquence curieuse d'une telle situation fut - pendant les mois qui suivirent immédiatement l'Indépendance - le succès inattendu d'un film documentaire qui avait été commandé par un important industriel indien (Scindia) pour mettre en valeur ses chantiers navals, film qui avait été tourné par P. V. Pathy, l'ancien élève de Sylvain Lévi en collaboration avec un autre cinéaste qui s'appelait Paul Zils. Le film s'intitulait India's Struggle for National Shipping et son succès était dû au fait qu'il comportait une importante compilation d'images d'actualité sur le Mouvement nationaliste et sur ses leaders, des années 20 jusqu’à l'Indépendance.

Il est paradoxal que ce soit un cinéaste allemand qui devint, pendant une décennie au moins, un des plus connus sinon le plus connu des auteurs de films documentaires dans l' Inde indépendante. Paul Zils avait alors l'habitude de se présenter comme un réfugié allemand ayant fui le nazisme pendant les années 30. Il était passé par Hollywood puis avait fait quelques films dans différents pays d'Asie avant de se retrouver en Indonésie au cours des années 40, où il y aurait été interné après l'invasion de la Hollande par l'Allemagne. Lorsque la menace japonaise s'est précisée, il semble qu'il ait été évacué d’ Indonésie sur un navire qui a été coulé par un sous-marin. Rescapé par un bateau de la flotte 
indienne, Zils a été de nouveau interné, en Inde cette fois, pendant toute la durée de la Seconde Guerre mondiale, comme l'étaient tous les citoyens allemands se tro u vant dans un territoire sous contrôle britannique.

En 1946, cependant, Zils recommença à travailler comme cinéaste en Inde, dabord pour les Anglais dans le cadre de l' Information Film of India pendant les quelques mois qui précédèrent sa dissolution, puis durant une douzaine d'années dans le cadre de l'Inde indépendante. Il fit de nombreux films en tant qu'indépendant; ses commandes émanaient aussi bien du nouveau gouvernement indien que de services de coopération étrangers, d'institutions internationales comme l'Unesco ou encore dentreprises multinationales, la Shell par e xemple. Pendant toute cette période, Zils fut considéré comme l'une des personnalités les plus importantes du cinéma documentaire à Bombay. Il fut aussi pendant quelques années l'éditeur en chef du seul magazine indien entièrement consacré à ce domaine (Indian Documentary) et il obtint plusieurs prix pour ses films dans des concours internationaux. À cette époque, il était souvent fait référence à ses films quand il s'agissait de donner les pre u ves de l'émergence dưne nouvelle tradition de films documentaires de qualité dans le tiers-monde. En 1958, il revint en Allemagne de l' Ouest, où il créa sa pro p recompagnie de films tout en travaillant comme consultant pour divers pays en voie de développement dans le cadre de la coopération ouest-allemande - et en particulier au Sri Lanka. On lui demandait de favoriser l'émergence d'une tradition nationale de cinéma documentaire dans ces différents pays.

Étant donné les connotations associées à ce nom dans les années 30 et la manière dont Zils s'était toujours fait passé pour un réfugié politique, il est surprenant qu'il ait appelé sa compagnie Condor Film Productions. Cela m’a conduit à examiner sa biographie d'un peu plus près. Encore en 1977, le directeur de l' Institut culturel allemand à Sri Lanka avait organisé une rétrospective de ses films en précisant que le réalisateur avait été formé " comme un jeune cinéaste durant l'Âge d'or du cinéma allemand avant la Seconde Guerre mondiale " ${ }^{31}$. Cet Âge d'or est celui qui correspond à la période où le cinéma allemand était passé sous le contrôle exclusif des nazis qui, par le biais des jeunesses hitlériennes, avaient aussi pris en charge la formation de très jeunes cinéastes.

Dans un des derniers entretiens qu'il eut l'occasion de donner vers la fin de sa vie, Zils se fit un peu plus prolixesur son passé. Effectivement formé au cinéma sous l'hitlérisme, puisqu'il avait fait son apprentissage de metteur en scène aux studios de Neubabelsberg en 1933, il s'y flatte, en particulier, de la relation privilégiée qu'il aurait eu avec Goebbels alors qu'il travaillait à l'UFA ${ }^{32}$. Ce qui demeure obscur cependant - étant donné les déclarations vagues de Zils à ce sujet - ce sont les raisons qui l'ont poussé à fuir le régime nazi. Cela rend difficile toute reconstitution précise de son itinéraire personnel. Mais quelle qu'ait pu en être la raison véritable, ce qui est plus significatif c'est la manière dont un cinéaste

31. D. Greiner, Paul Zils Cine Creations, Sri Lanka, German Cultural Institute, 1977.

32. E. Barnouw, Documentary ..., op. cit., 1974 : 166-167. 
comme lui a pu, au cours de sa carrière, mettre ses compétences au service de patrons aussi différents les uns des autres. Il y a une certaine ironie, en effet, à découvrir que l'homme que l'on voit en train de sourire à Nehru sur une photo dédicacée par ce dernier lors de la présentation de son film de fiction Hindustan hamara ("Notre Inde») soit le même qui a successivement été au service des nazis puis de l'Empire britannique avant dêt tre finalement financé par la République ouest-allemande.

Mais là encore, le cas de Paul Zils serait assez peu intéressant s'il était unique. Tel n'est cependant pas le cas et, pour en donner un autre exemple, j'ai été personnellement surpris d'apprendre - même si c'est un fait connu des cinéphiles avertis - que Rossellini, avant de devenir le cinéaste respecté de Rome, ville ouvente, avait aussi tourné plusieurs films de propagande à la gloire de l'armée fasciste italienne; l'un d’entre eux d'ailleurs à partir d'un scénario du fils de Mussolini, et avec l'assistance d'Antonioni.

De tels cas viennent ainsi confirmer ce qui semble avoir été un trait constant du cinéma documentaire entre les années 20 et 60 , à savoir la manière dont son émergence et son développement ont transcendé - comme on l'a vu auparavant toutes les frontières, idéologiques, culturelles ou nationales. Mais c'est aussi l'art consommé avec lequel un nombre restreint de cinéastes sont parvenus à s'imposer à travers le monde en n'hésitant pas à mobiliser toutes les formes de patronage qui s'offraient à eux, indépendamment là encore des barrières culturelle, nationale ou idéologique.

\section{Le paradigme perdu}

L'audace véritablement anthropologique de Warburg aura été de questionner, avec une fraîcheur renouvelée, ce qui était la dimension non seulement la mieux connue mais aussi la plus caractéristique de la Renaissance : à savoir l'influence exercée par des modèles de l'Antiquité sur les arts visuels à cette époque.

Cela l'a conduit à découvrir en particulier que des formes de représentation qui étaient imputées au regard nouveau que les artistes de la Renaissance posaient sur les gens et sur les choses avaient en fait été directement inspirées par des modèles empruntés à l'Antiquité. De même chercha-t-il à montrer que des motifs allégoriques que l'on aurait pu attribuer à la seule imagination des a rtistes de cette période pouvaient re n voyer à des origines lointaines et variées (dont l' Inde en particulier) ${ }^{33}$. C'est aussi cette recherche qui inspira à ce dernier la formule laconique et magnifique par laquelle il conclut l'un de ses essais en se référant à cette "époque de migration internationale des images que nous appe-

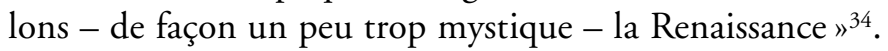

33. Aby Warburg, "Art italien et astrologie internationale au Palazzo di Schifanoia à Ferrare », in Essais florentins, Paris, Klincksieck, 1990 : 200-216.

34. Ibid. : 216. 
Cet aspect de l'œuvre de Warburg a été développé et enrichi par ses successeurs, non seulement sur le plan des faits par des érudits comme Rudolf Wittkower ${ }^{35}$, mais aussi sur le plan épistémologique. Il y a, en particulier, dans l'œuvre d'Ernst Gombrich, des pages magnifiques sur les nouvelles perspectives qui s'offrent à l'histoire culturelle dès lors qu'on tire des conclusions rigoureuses de la substitution méthodologique de la notion de "mouvement " à celle de "période " ${ }^{36}$.

Aussi peut-il sembler que Warburg n'ait pas eu, en fin de compte, nécessairement tort quand il considérait qu'une telle inspiration pourrait être également d'un grand profit pour la compréhension d'un phénomène apparemment aussi éloigné de l'art de la Renaissance que pouvait l'êt rela propagande au XXe siècle. Dans les pages qui précèdent, j'ai cherché à montrer le bénéfice que l'on peut tirer à s'inspirer, pour l'étude de films documentaires, de la fraîcheur de regard dont Warburg avait fait pre uve vis-à-vis de l' a rt de la Renaissance.

Étant donné, en particulier, l'importance des déterminations institutionnelles, commerciales ou politiques qui ont toujours lourdement pesé sur la production de documentaires, l'étonnant n'est pas, en effet, que de telles contraintes aient pu, dans une certaine mesure, déterminer le contenu comme le style de ces films, c'est plutôt que celles-ci les aient, tout compte fait, aussi peu déterminés. Autant on dispose de tentatives très élaborées (et de débats persistants) pour évaluer exactement le degré de l'autonomie de l' a rtvis-à-vis du contexte qui est le sien ${ }^{37}$, autant, il peut sembler que la même question soit souvent tranchée - avant même qu'on l'ait posée - dans le cas des arts proches de la propagande. Supposons cependant que l'on constate une bien moindre influence des commanditaires sur les créateurs et des œuvres sur leur audience que celle à laquelle on s'attendrait dans le cas de la propagande où de telles déterminations sont censées s'exprimer dans toute leur force! Cela oblige, du même coup à réviser considérablement la portée conceptuelle des analyses qui sont faites dans les cas opposés où les manifestations culturelles sont communément identifiées à de l'a rt " pur ".

De même, personne n'ignore véritablement l'internationalisme des avantgardes artistiques au XXe siècle, ne serait-ce qu'en raison des attaques dont cellesci ont été l'objet de la part des idéologies totalitaires. Mais peut-être n'a-t-on pas suffisamment souligné que des formes d'a rt ou d'activités culturelles qui s'affichaient à la même époque comme des traditions purement "nationales » et dont l'un des traits récurrents était leur hostilité déclarée face à toute forme de cosmopolitisme étaient loin de pouvoir être réduites elles-mêmes, sinon de manière tout à fait superficielle, à leurs seules spécificités culturelles ou nationales. Tel est le cas, en particulier, en ce qui concerne le cinéma documentaire.

Il est difficile, en effet, de tro u ver aucun autre domaine d'activité culturelle qui se caractérise à un tel degré non seulement par une «circulation internatio-

35. Rudolf Wittkower, L'Orient fabuleux, Paris, Thames \& Hudson, 1991.

36. Ernst Gombrich, Ideals and Idols, Oxford, Phaidon Press, 1979: 50-51.

37. Voir en particulier Pierre Bourdieu, Les Règles de l'art, Paris, Le Seuil, 1998. 
nale" systématique des images, des styles, et des contenus, mais aussi par une telle capacité des réalisateurs à passer, comme ils l'ont fait, d'une cause à l'autre, 264 d'idéologies en idéologies, de pays en pays, de patrons en patrons. C'est d'ailleurs cette capacité, bien plus que l'adéquation des films documentaires au contexte du moment, qui explique la manière dont une telle tradition a pu se développer et se diffuser bien au-delà des idéologies ou des circonstances auxquelles elle a pu servir temporairement damplificateur.

Mais cela veut-il dire pour autant que la capacité du cinéma documentaire à s'imposer plus ou moins dans le monde entier, presque indépendamment du contexte précis où il est produit, témoigne également du caractère plus ou moins « universel » des ressorts esthétiques ou psychologiques sur lesquels il fonderait son pouvoir de séduction? Si tel était le cas, cette explication renverrait, de fait, à un autre versant important de l'œuvre de Warburg.

Celui-ci a longtemps cherché - comme on le sait - à fonder sa conception de l'art et de son histoire sur l'impact psychologique et émotionnel durable de certaines images. Car c'est précisément, aux yeux de Warburg, la manière dont leur puissance émotionnelle peut toujours être réactivée qui explique aussi la manière dont certains types d'images ne cessent de connaittre une nouvelle «vie" dans les contextes et les circonstances les plus variés. Comment ne pas noter dès lors qu'une telle conception de l'esthétique ne fait pas seulement écho aux préoccupations des praticiens de la propagande, mais peut aussi offrir une assise théorique à leur activité ?

Toujours dans la même perspective, il est également curieux de constater que Gombrich, habituellement plus réservé quant au versant psychologique de l'œuvre de Warburg, semble pourtant entériner une telle perspective dans son analyse de la propagande. Selon celui-ci, en effet, la propagande nazie à la radio devrait son influence à la manière dont elle serait à même de faire écho aux tendances paranoïaques qui existent chez chacun d'entre nous. Inutile de le préciser, cependant, une telle explication trouve rapidement ses limites. Ainsi pour prendre un simple exemple, que j'emprunterai, cette fois encore, au sous-continent indien, c'est un fait avéré que la propagande nazie à la radio a trouvé une certaine audience dans les milieux éduqués de la région pendant les premiers temps de la Seconde Guerre mondiale. Toutefois, un tel impact n'avait rien à voir avec la paranoïa de quiconque, sa cause était due à l'espoir entretenu par certains nationalistes de l'époque de voir l'Angleterre relâcher son emprise si elle était défaite par l'Allemagne.

Aussi j'aimerais conclure cet essai en suggérant que si le cinéma documentaire a rencontré un tel succès à travers le monde, ce n'est probablement pas dû au fait qu'il a correspondu aux attentes spécifiques de ses commanditaires ou de ses audiences, mais ce n'est probablement pas non plus parce qu'il a véhiculé des images dont l'impact aurait touché en profondeur la psychologie de chacun. D'ailleurs la majorité des historiens qui s'intéressent de près à la propagande semblent penser qu'elle n'a probablement pas eu l'influence qu'on a eu tendance à lui prêter à l'époque où elle fut largement utilisée. Mais quelle que soit la 
vérité, il n'y a pas de doute que la croyance en l'existence d'un impact décisif des images sur le plan psychologique a constitué un facteur essentiel pour expliquer l'importance attribuée à la propagande et la rapidité avec laquelle l'usage s'en est répandu dans le monde au cours du siècle dernier. Et c'est précisément cette croyance que l'on ret ro uve en particulier chez Warburg. Aussi, le fait qu'il n'ait jamais réussi à articuler une théorie satisfaisante de la puissance émotive qu'il prêtait aux images a probablement été pour lui un terrible drame épistémologique. Mais pour nous, il valait probablement mieux qu'il en fût ainsi.

MOTS CLÉS/KEYWORDS : histoire de l'art/art history - propagande/propaganda - cinéma documentaire/documentary films - Aby Warburg - John Grierson - Empire britannique/British empire - Ernst Gombrich - Inde/India.

RÉSUMÉ/ABSTRACT

Denis Vidal, La migration des images. Histoire de l'art et cinéma documentaire. - La propagande et l'histoire de l'art ne sont pas toujours des activités aussi éloignées l'une de l'autre qu'on pourrait le penser. On sait d'ailleurs qu'Aby Warburg et bien d'autres historiens de l'art (Émile Mâle, Ernst Gombricht, Kenneth Clark, en particulier) n'ont pas vraiment hésité à mettre leurs connaissances au service de la propagande de leurs pays respectifs quand les circonstances l'exigeaient. Mais plus fondamentalement aussi, les problèmes que pose l'analyse de la propagande obligent à s'interroger sur certains des postulats les plus centraux de l'histoire de l'art. C'est ce qui est montré ici, en présentant plus spécifiquement les débuts du cinéma documentaire dans l'Empire britannique et en Inde.
Denis Vidal, The Migration of Images: The History of Art and Film Documentaries. Propaganda and art history do not lie as far apart as we might think. As we know, Aby Warburg and many other art historians (such as Émile Mâle, Ernst Gombricht or Kenneth Clark) have not hesitated to put their knowledge in the service of propaganda for their homelands when circumstances required doing so. At a more fundamental level, the problems of analyzing propaganda force us to investigate some of art history's key postulates. This is shown by focusing on the very beginnings of documentary films in the British Empire and India. 
